\title{
Evaluation of Anticoagulation Control among Patients Taking Warfarin in University of Gondar Hospital, Northwest Ethiopia
}

\author{
Zelalem Liyew, Abilo Tadesse $(\mathbb{D}$, Nebiyu Bekele, and Tewodros Tsegaye \\ Department of Internal Medicine, School of Medicine, College of Medicine and Health Sciences, University of Gondar, \\ Gondar, Ethiopia \\ Correspondence should be addressed to Abilo Tadesse; abilo.tadesse@yahoo.com
}

Received 9 August 2021; Revised 25 October 2021; Accepted 9 December 2021; Published 21 December 2021

Academic Editor: Elvira Grandone

Copyright $(92021$ Zelalem Liyew et al. This is an open access article distributed under the Creative Commons Attribution License, which permits unrestricted use, distribution, and reproduction in any medium, provided the original work is properly cited.

Introduction. Warfarin is a widely used oral anticoagulant in clinical practice. It has variable intraindividual and interindividual dose response and a narrow therapeutic index. Therefore, it requires frequent and regular international normalized ratio (INR) determination to maintain the INR within the therapeutic range. The study evaluated parameters of anticoagulation control among patients on warfarin. Methods. A cross-sectional study was conducted at University of Gondar hospital. A consecutive sampling method was used to recruit study subjects. The anticoagulation control was evaluated by determining the proportion of desired INRs and the proportion of time spent in the therapeutic range (TTR). Logistic regression analysis was used to identify associated factors with adequate TTR. A $P$ value $<0.05$ was used to declare significant association. Result. A total of 338 study subjects were included in the study. The mean age of patients was 48.8 ( $\mathrm{SD}=16.4$ ) years. Atrial fibrillation was the commonest indication for warfarin therapy. One-third (33\%) of study subjects achieved the desired INRs of 2.0-3.0, while about one-tenth $(13 \%)$ of patients attained good INR control (TTR $\geq 65 \%)$. Multivariate logistic regression analysis revealed no significant association of sociodemographic and clinical characteristics with good TTR outcome. Conclusion. The level of anticoagulation control with warfarin among study subjects was very low. The authors recommend to implement a validated warfarin-dose titration protocol and to establish anticoagulation clinics to mitigate the low anticoagulation level.

\section{Introduction}

Warfarin is the widely prescribed oral anticoagulant in clinical practice. It is used to prevent and treat arterial emboli in patients with atrial fibrillation and treat deep venous thrombosis [1-4]. Warfarin is a challenging drug which has variable intraindividual and interindividual dose response and a narrow therapeutic index. Therefore, it requires frequent and regular INR monitoring to maintain the INR within the therapeutic range $[3,4]$. The proportion of INRs within the range of 2.0-3.0 (INR $=2.5-3.5$ in prosthetic heart valves) determines the desired INR value. Therapeutic efficacy of warfarin will be reduced when INR value $<2.0$ and intrinsically lost when INR value $<1.5$ [3-7]. The proportion of time spent in the therapeutic INR range (TTR) evaluates the quality of anticoagulation control [3-7]. TTR $\geq 65 \%$ declares "good" INR control. TTR $<65 \%$ confirms reduced warfarin efficacy and increased risk of thromboembolic events or bleeding episodes. TTR $<40 \%$ notifies loss of survival benefit with warfarin use [7-11]. Sub-Saharan African studies documented that proportions of desired INR value $=2.0-3.0$ and TTR $\geq 65 \%$ were $30-40 \%$ and $15-25 \%$, respectively [12-16]. Global studies documented that old age, obese individuals, other concomitant drugs intake, excessive alcohol intake, and renal or hepatic dysfunctions were among the listed causes of poor TTR outcome $($ TTR $<65 \%)$ [8-11, 17-21]. Despite the wide availability of warfarin as an oral anticoagulant, information on its level of anticoagulation control is scarce in Ethiopia. This study aimed to determine the magnitude of proportion of INRs within the desired range, proportion of time spent in the therapeutic INR range, and factors associated with quality of anticoagulation outcomes in patients taking warfarin in a hospital setting, in Northwest Ethiopia. 


\section{Methods}

2.1. Study Setting. A hospital-based cross-sectional study was conducted between November 1, 2019, and October 31, 2020, at Cardiology and Hematology Clinics, University of Gondar hospital, Northwest Ethiopia. The Cardiology and Hematology units provide healthcare services for outpatients who were followed up at the clinics. Both clinics were run by internists, medical residents, and respective unit nurses. Patients were admitted every one to three months based on their severity of illness.

2.2. Study Population and Study Subjects. Patients older than 18 years and who were on warfarin with follow-up at Cardiology and Hematology Clinics, University of Gondar hospital, during the study period were the study population.

2.3. Inclusion Criteria. Patients aged 18 years old and above, who were on warfarin for at least 6 months, had at least 6 INR determinations, and had each consecutive INR determination less than 2 months apart were included in the study.

2.4. Exclusion Criteria. Patients who did not give consent to the study, refused to be included in the study, and had incomplete data were excluded from the study.

2.5. Study Variables. Dependent variables included the proportion of time spent in the therapeutic INR range (TTR).

Independent variables included the following: (1) sociodemographic characteristics including age, gender, occupation, marital status, educational level, income level, residence, and religion; (2) clinical characteristics including indication for warfarin, warfarin dosage, concomitant drugs intake (antiplatelets, statins, antihypertensive drugs, antithyroid drugs, antiretroviral drugs), adherence to warfarin, INR monitoring interval, co-existing comorbidities, body mass index, and alcohol intake.

2.6. Sample Size and Sampling Procedure. The sample size was calculated using a single population proportion formula with the assumption of 95\% confidence level and 5\% margin of error and taking 30\% for TTR in the sub-Saharan African region [12]. A consecutive sampling method was used to recruit 338 study subjects.

2.7. Data Collection Instrument and Procedures. Data were collected through an investigator-administered predesigned questionnaire. The questionnaire was prepared in English and translated into local language (Amharic) for data collection and then retranslated back to English while maintaining its consistency. The questionnaire had been pretested on 34 patients in a similar setup before the actual data collection was commenced to check for consistency and reliability of the questionnaire. Patients were interviewed to obtain sociodemographic data. Relevant medical history and laboratory parameters were obtained from patients' records. One supervisor $(\mathrm{MD}+)$ and two data collectors (MD) participated in the data collection process. Quality of data was ensured through training and supervision of data collectors.

2.8. Data Analysis. Data were entered into EPI Info version 4.4.1 and transported to SPSS version 20 for analysis.

Patient characteristics were reported as counts (percentages) for categorical variables and mean with standard deviation for continuous variables. Bivariate and multivariate logistic regression models were constructed to identify associated factors with anticoagulation outcome. Crude odds ratio (COR) and adjusted odds ratio (AOR) were reported. A $P$ value $<0.05$ was used to declare a significant association.

2.9. Ethical Considerations. The research protocol complied with the Declaration of Helsinki, and ethical clearance was obtained from the Institutional Review Board (IRB) of College of Medicine and Health Sciences, University of Gondar (04/09/2012; IRB No. 09/20/627/12). Study subjects were recruited only after informed written consent was obtained. All data obtained were treated confidentially. During the data collection process, those patients who were found to have atrial fibrillation and venous thromboembolism were taken care of as per the recommendations of AHA/ACC guidelines and American Society of Hematology guideline, respectively $[2,3]$.

2.10. Definition of Terms. The proportion of INRs within the desired range of 2.0-3.0 (2.5-3.5 for mechanical prosthetic valves) is defined as the number of INRs within the target range divided by the number of INR measurements per patient [22].

The proportion of time spent within the therapeutic INR range (TTR) is defined as the duration of time for which the patient's international normalized ratio (INR) values were within a therapeutic range of 2.0-3.0 (2.5-3.5 for mechanical prosthetic valves). It was calculated using Rosendaal's method, which has used linear interpolation to assign an INR value to each day between successive observed INR values. TTR was calculated as the number of person-days with therapeutic INR range divided by the total number of person-days on warfarin. TTR $\geq 65 \%$ declares "good" INR control, while TTR $<65 \%$ affirms "poor" INR control [23].

Warfarin adherence: "continuous, single interval measure of medication gaps" was used to assess medication refill for warfarin. It was calculated as the sum of the days a patient was late for warfarin pick-up appointments in each month of the year, divided by the total number of days between pickup periods in the year of study. Nonadherence was defined as more than one-third of days late for warfarin pick-up appointments [24].

Major bleeding in nonsurgical patients is defined as fatal bleeding; and/or symptomatic bleeding in a critical area or organ, such as intracranial, intraspinal, intraocular, 
retroperitoneal, intra-articular or pericardial, or intramuscular with compartment syndrome; and/or bleeding causing a fall in hemoglobin of $2 \mathrm{gm} / \mathrm{dl}$ or more, or leading to transfusion of 2 or more units of whole blood or red cells [25].

Clinically relevant nonmajor bleeding in nonsurgical patients is defined as any sign or symptom of hemorrhage (e.g., more bleeding than would be expected for a clinical circumstance, including bleeding found by imaging alone) that does not fit the criteria for the ISTH definition of major bleeding but does meet at least one of the following criteria: requiring medical intervention by a healthcare professional; leading to hospitalization or increased level of care; prompting a face-to-face (i.e., not just a telephone or electronic communication) evaluation [26].

\section{Results}

3.1. Sociodemographic Characteristics of Study Participants. A total of 338 patients on warfarin were included in the study. The mean age of study subjects was $48.8(\mathrm{SD}=16.4)$ years. The majority of study participants were females (217/ $338,64 \%)$, married $(247 / 338,73 \%)$, and urban dwellers (193/ 338, 57\%). Most respondents were Christian by religion $(290 / 338,86 \%)$, and half $(164 / 338,49 \%)$ of them attended formal education (Table 1).

3.2. Clinical Characteristics of Study Participants. Three-quarters $(252 / 338,75 \%)$ of patients received warfarin for atrial fibrillation (Figure 1). More than half (199/338, $59 \%$ ) of patients were taking warfarin dose $<5 \mathrm{mg}$, PO daily. Most patients $(305 / 338,90 \%)$ received other concomitant drugs like antihypertensive drugs (102/338, 30\%), antiplatelets $(144 / 338,43 \%)$, lipid-lowering drugs (statins) (75/ $338,22 \%)$, antithyroid drugs (PTU) $(51 / 338,15 \%)$, and antiretroviral drugs (ART) $(22 / 338,7 \%)$. Most patients $(283 /$ $338,84 \%$ ) had INR determination every month. Heart failure and hyperthyroidism were detected in 144/338 (43\%) and $46 / 338$ (15\%) patients, respectively. Most patients (277/ $338,82 \%)$ never touched alcohol (Table 2).

3.3. Proportion of INRs within the Desired Range. Among 334 patients with atrial fibrillation, venous thromboembolism, and intracardiac thrombus, the proportion of desired INRs was determined. One-third $(110 / 334,33 \%)$ of patients achieved therapeutic INR range (INR $=2.0-3.0)$. However, half $(165 / 334,49 \%)$ of the patients achieved subtherapeutic INR range $(\mathrm{INR}<2.0)$. The supratherapeutic INR range (INR > 3.0) was attained by less than one-fifth $(59 / 334,18 \%)$ of patients. Among 4 patients with mechanical prosthetic valves, only one patient $(25 \%)$ attained the desired INR range $(\mathrm{INR}=2.5-3.5)$, while the remaining, 3/4 $(75 \%)$, achieved the subtherapeutic INR range $(\mathrm{INR}<2.5)$.

3.4. Proportion of Time Spent in the Therapeutic INR Ranges. The proportion of time spent within the therapeutic range (TTR) was determined for all study participants (Figure 2). More than one-tenth $(44 / 338,13 \%)$ of patients achieved
TABLE 1: Sociodemographic characteristics of patients taking warfarin in University of Gondar hospital, Northwest Ethiopia, from November 1, 2019, to October 31, $2020(n=338)$.

\begin{tabular}{lcc}
\hline Variables & Frequency (no.) & Percentage \\
\hline Age (years) & & \\
$\quad$ <40 years & 115 & 34.0 \\
40-60 years & 110 & 32.5 \\
$\quad>60$ years & 113 & 33.5 \\
Gender & 121 & \\
$\quad$ Male & 217 & 35.8 \\
$\quad$ Female & & 64.2 \\
Residence & 193 & \\
$\quad$ Urban & 145 & 57.1 \\
$\quad$ Rural & & 42.9 \\
Educational level & 71 & \\
$\quad$ Cannot read and write & 103 & 21.0 \\
Informal education & 51 & 30.5 \\
$\quad$ Elementary school & 64 & 15.1 \\
Secondary school & 49 & 18.9 \\
College and above & & 14.5 \\
Monthly income (in birr) & 183 & 54.1 \\
$\quad<1500$ & 85 & 25.1 \\
$\quad$ 1500-3000 & 70 & 20.8 \\
$\quad>3000$ &
\end{tabular}

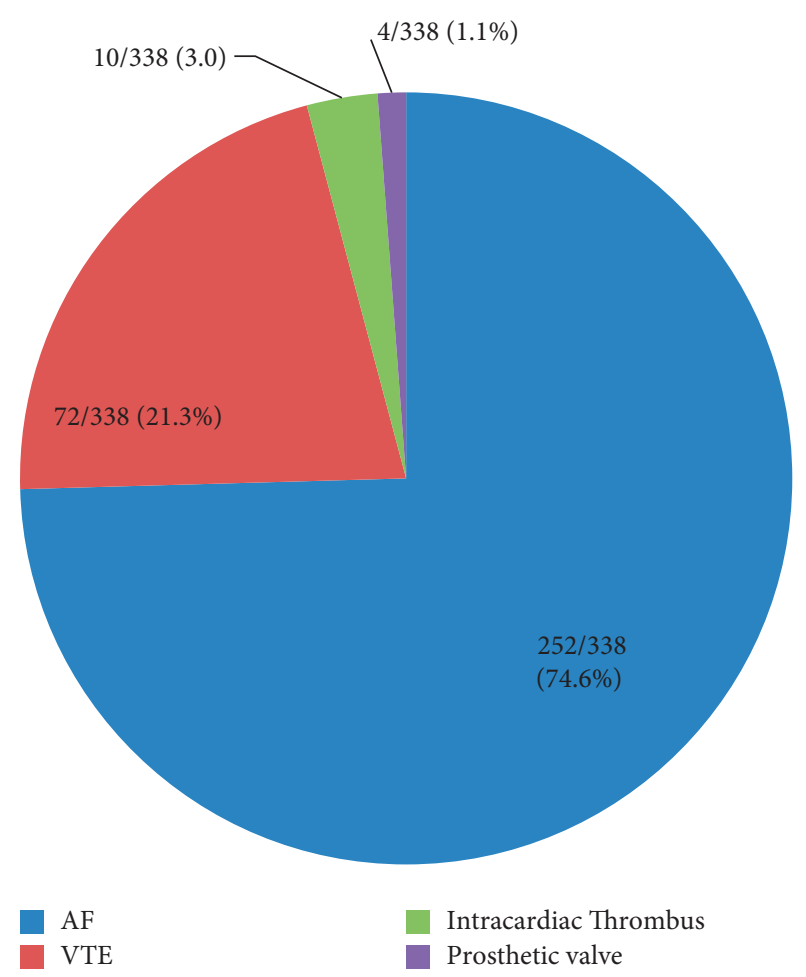

FIGURe 1: Pie chart showing number (percentage) of indications for warfarin therapy. AF, atrial fibrillation; VTE, venous thromboembolism.

good INR control (TTR $\geq 65 \%$ ). More than one-third (125/ $338,37 \%)$ of study subjects had poor TTR control (TTR $=36-65 \%)$. Half $(169 / 338)$ of the study subjects attained TTR $<35 \%$, which was interpreted as no survival benefit from warfarin therapy. 
TABLE 2: Clinical characteristics of patients taking warfarin in University of Gondar hospital, Northwest Ethiopia, from November 1, 2019, to October 31, $2020(n=338)$.

\begin{tabular}{|c|c|c|}
\hline Clinical characteristics & Frequency (no.) & Percentage \\
\hline \multicolumn{3}{|l|}{ BMI $\left(\mathrm{kg} / \mathrm{m}^{2}\right)$} \\
\hline$<18.5^{\circ}$ & 29 & 8.6 \\
\hline $18.5-24.9$ & 229 & 67.7 \\
\hline $25.0-29.9$ & 53 & 15.7 \\
\hline$\geq 30$ & 27 & 8.0 \\
\hline \multicolumn{3}{|l|}{ Indication for warfarin } \\
\hline Atrial fibrillation & 252 & 74.6 \\
\hline Venous thromboembolism & 72 & 21.3 \\
\hline Prosthetic heart valves & 4 & 1.1 \\
\hline Intracardiac thrombus & 10 & 3.0 \\
\hline \multicolumn{3}{|l|}{ Dose of warfarin } \\
\hline$<5 \mathrm{mg}$, daily & 199 & 58.9 \\
\hline $5 \mathrm{mg}$, daily & 118 & 34.9 \\
\hline$>5 \mathrm{mg}$, daily & 21 & 6.2 \\
\hline \multicolumn{3}{|l|}{ INR monitoring interval } \\
\hline Every 2 weeks & 32 & 9.5 \\
\hline Every 1 month & 283 & 83.7 \\
\hline Every 2 months & 23 & 6.8 \\
\hline \multicolumn{3}{|l|}{ Nonadherence to warfarin } \\
\hline Yes & 82 & 24.3 \\
\hline \multicolumn{3}{|l|}{ Concomitant drugs use } \\
\hline Yes & 305 & 90.2 \\
\hline
\end{tabular}

BMI, body mass index; concomitant drugs use included antiplatelets, statins, antihypertensives, antithyroid drugs, and antiretroviral drugs.

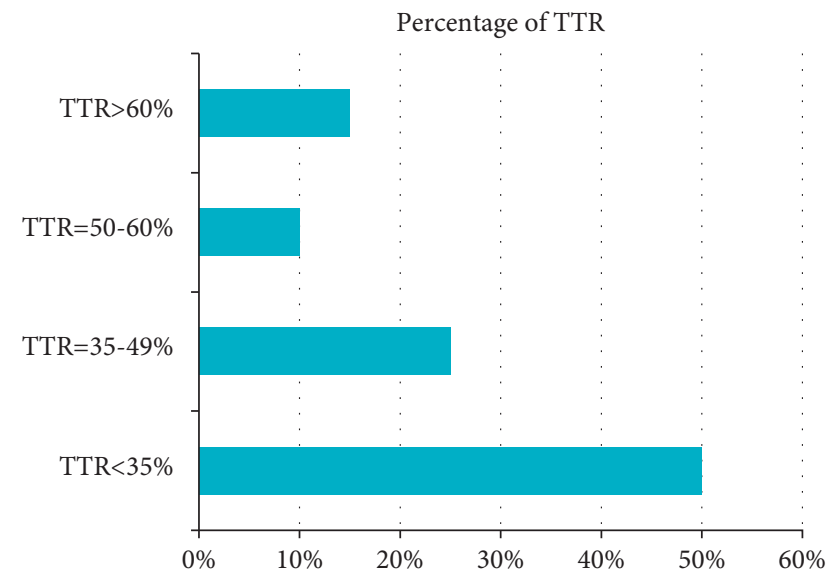

FIGURE 2: Bar diagram showing the percentage of TTR among patients on warfarin.

3.5. Bleeding Events among Patients on Warfarin. A quarter $(85 / 338,25 \%)$ of patients on warfarin experienced clinically relevant nonmajor bleeding; this had required weekly INR determination and dose adjustment. Among patients with nonmajor bleeding episodes, almost all (77/85, 90\%) had TTR $<65 \%$ and the remaining $(8 / 85,10 \%)$ had TTR $\geq 65 \%$. No major bleeding episodes or vascular thrombosis were noticed among patients on warfarin.

3.6. Factors Associated with Anticoagulation Control. On bivariate analysis, VTE as indication for warfarin; warfarin dose, $5 \mathrm{mg}$ PO daily; and absence of heart failure were predictors of good INR outcome (TTR $\geq 65 \%$ ). None of the above variables were found to be significant when regressed on multivariate analysis. Bivariate analysis revealed no significant association of sociodemographic characteristics including age, gender, educational level, and monthly income with good INR outcome (TTR $\geq 65 \%$ ). Similarly, clinical characteristics including dose of warfarin, warfarin adherence, INR monitoring interval, other concomitant drugs intake, other comorbidities, body mass index, and alcohol intake showed no significant association with good INR outcome (TTR $\geq 65 \%$ ) (Table 3 ).

\section{Discussion}

Atrial fibrillation (75\%) was the commonest indication for warfarin use, followed by venous thromboembolism and prosthetic heart valves. This finding was shared by reports from other sub-Saharan African countries [12-16]. The proportion of adequate anticoagulation outcome (TTR $\geq 65 \%$ ) in the study subjects was $13 \%$. The finding was on the lower margin of previous reports in sub-Saharan Africa, where the proportion of adequate INR level was 15-25\% [12-14]. However, the United States and European studies have shown that the proportion of adequate anticoagulation level was 50-70\% [7-11]. The reason for varied magnitude of anticoagulation control among countries was partly explained by their difference in anticoagulation management services. Reports from the Western world had shown that establishing anticoagulation clinics, implementing computer-assisted warfarin dosing, self-testing and self-management among motivated patients, and instituting coordinated follow-up clinics had improved the anticoagulation control $[27,28]$. In this study, only one-third (33\%) of study subjects attained the desired INR value (INR $=2.0-3.0)$ and majority (59\%) were on low-dose warfarin ( $<5 \mathrm{mg}$, daily). Physicians might tend to undertreat patients because of fear of bleeding [29]. A quarter (24\%) of patients were nonadherent to their medication. Patients' comprehension on health benefits of the oral anticoagulant might be low [30]. There was no validated warfarin-dose titration protocol in the hospital. Warfarin-dose titration was based on conventional clinical practice. "Anticoagulation clinic" for patients' close follow-up was not available. These challenging conditions might have contributed to poor anticoagulation control. On bivariate analysis, VTE as indication for warfarin; warfarin dose, $5 \mathrm{mg}$ PO daily; and absence of heart failure were predictors of good INR outcome (TTR $\geq 65 \%$ ). Patients with VTE might not have pill fatigue, since time to treatment was limited. Even though patients on higher warfarin dosage might achieve an acceptable INR target, variant alleles of the CYP2C9 and VKORC1 genes among patients might affect the warfarin-dose requirements, sensitivity to warfarin, and quality of anticoagulation. Few studies documented the presence of genetic polymorphisms to warfarin among African descents [31]. Patients with no heart failure might not be on multiple drugs, which then limited drug-drug interactions. On multivariate analysis, explanatory sociodemographic and clinical characteristics showed no 
TABLE 3: Bivariate and multivariate logistic regression analyses of time spent within the therapeutic INR range (TTR) among patients taking warfarin in University of Gondar, Northwest Ethiopia, from November 1, 2019, to October 31, $2020(n=338)$.

\begin{tabular}{|c|c|c|c|c|c|c|}
\hline \multirow{2}{*}{ Variables } & \multicolumn{2}{|c|}{ TTR } & \multirow{2}{*}{$\mathrm{COR}(\mathrm{CI})$} & \multirow{2}{*}{$P$ value } & \multirow{2}{*}{$\mathrm{AOR}(\mathrm{CI})$} & \multirow{2}{*}{$P$ value } \\
\hline & TTR $<65 \%$ & $\mathrm{TTR} \geq 65 \%$ & & & & \\
\hline \multicolumn{7}{|l|}{ Age } \\
\hline$<40$ years & 100 & 15 & $1.04(0.48,2.27)$ & 0.922 & & \\
\hline $40-60$ years & 97 & 13 & $0.94(0.42,2.12)$ & 0.877 & & \\
\hline$>60$ years & 99 & 14 & 1 & & & \\
\hline \multicolumn{7}{|l|}{ Gender } \\
\hline Male & 103 & 18 & 1 & & & \\
\hline Female & 193 & 24 & $1.41(0.73,2.71)$ & 0.310 & & \\
\hline \multicolumn{7}{|l|}{ Marital status } \\
\hline Single & 50 & 11 & 1 & & & \\
\hline Married & 218 & 28 & $1.98(0.51,7.71)$ & 0.325 & & \\
\hline Others & 27 & 3 & $1.15(0.33,4.04)$ & 0.825 & & \\
\hline \multicolumn{7}{|l|}{ Monthly income } \\
\hline$<1500$ birr & 157 & 26 & 1 & & & \\
\hline 1500-3000 birr & 77 & 8 & $1.28(0.55,2.99)$ & 0.563 & & \\
\hline$>3000$ birr & 62 & 8 & $0.81(0.29,2.27)$ & 0.682 & & \\
\hline \multicolumn{7}{|l|}{ Educational level } \\
\hline Cannot read and write & 61 & 10 & 1 & & & \\
\hline Informal education & 91 & 12 & $1.80(0.53,6.12)$ & 0.345 & & \\
\hline Elementary school & 45 & 6 & $1.45(0.44,4.76)$ & 0.539 & & \\
\hline Secondary school & 54 & 10 & $1.47(0.39,5.56)$ & 0.573 & & \\
\hline College and above & 44 & 4 & $2.04(0.60,6.94)$ & 0.255 & & \\
\hline \multicolumn{7}{|l|}{ Residence } \\
\hline Urban & 170 & 23 & $0.89(0.47,1.72)$ & 0.744 & & \\
\hline \multirow{2}{*}{\multicolumn{7}{|c|}{ BMI $\left(\mathrm{kg} / \mathrm{m}^{2}\right)$}} \\
\hline & & & & & & \\
\hline$<18.5$ & 25 & 4 & 1 & & & \\
\hline $18.5-24.9$ & 199 & 30 & $1.44(0.39,5.19)$ & 0.578 & & \\
\hline$\geq 25$ & 72 & 8 & $1.36(0.59,3.09)$ & 0.469 & & \\
\hline \multicolumn{7}{|l|}{ Indication for warfarin } \\
\hline Atrial fibrillation & 222 & 30 & 1 & & & \\
\hline VTE & 64 & 8 & $0.14(0.02,0.99)$ & 0.049 & $0.56(0.14,2.18)$ & 0.404 \\
\hline Others & 10 & 4 & $0.31(0.08,1.23)$ & 0.097 & $0.43(0.09,1.94)$ & 0.271 \\
\hline \multicolumn{7}{|l|}{ Dose of warfarin } \\
\hline$<5$ mg daily & 181 & 18 & 1 & & & \\
\hline $5 \mathrm{mg}$ daily & 98 & 20 & $0.39(0.12,1.32)$ & 0.133 & $0.40(0.09,1.64)$ & 0.282 \\
\hline$>5 \mathrm{mg}$ daily & 17 & 4 & $0.91(0.28,2.98)$ & 0.877 & $0.94(0.24,3.74)$ & 0.950 \\
\hline \multicolumn{7}{|l|}{ Nonadherence to warfarin } \\
\hline Yes & 40 & 2 & 1 & & & \\
\hline No & 266 & 30 & $2.26(0.52,9.81)$ & 0.278 & & \\
\hline Concomitant drugs use & & & & & & \\
\hline Yes & 268 & 37 & 1 & & & \\
\hline No & 28 & 5 & $1,29(0.47,3.56)$ & 0.618 & & \\
\hline Frequency of INR monito & & & & & & \\
\hline Every 2 weeks & 28 & 4 & $0.68(0.15,3.05)$ & 0.613 & & \\
\hline Every 1 month & 249 & 34 & $0.65(0.21,2.02)$ & 0.455 & & \\
\hline Every 2 months & 19 & 4 & 1 & & & \\
\hline Alcohol intake & & & & & & \\
\hline Yes & 54 & 6 & 1 & & & \\
\hline No & 241 & 36 & $1.34(0.54,3.34)$ & 0.531 & & \\
\hline Chronic liver disease & & & & & & \\
\hline Yes & 14 & 3 & $0.91(0.46,1.80)$ & 0.791 & & \\
\hline No & 122 & 16 & 1 & & & \\
\hline Unknown & 160 & 23 & $1.49(0.39,5.59)$ & 0.554 & & \\
\hline Chronic kidney disease & & & & & & \\
\hline Yes & 25 & 4 & $1.32(0.62,2.85)$ & 0.473 & & \\
\hline No & 184 & 28 & 1 & & & \\
\hline Unknown & 87 & 10 & $1.39(0.40,4.82)$ & 0.602 & & \\
\hline
\end{tabular}


TABLE 3: Continued.

\begin{tabular}{|c|c|c|c|c|c|c|}
\hline \multirow{2}{*}{ Variables } & \multicolumn{2}{|c|}{ TTR } & \multirow{2}{*}{$\mathrm{COR}(\mathrm{CI})$} & \multirow{2}{*}{$P$ value } & \multirow{2}{*}{$\mathrm{AOR}(\mathrm{CI})$} & \multirow{2}{*}{$P$ value } \\
\hline & TTR $<65 \%$ & TTR $\geq 65 \%$ & & & & \\
\hline \multicolumn{7}{|c|}{ Heart failure } \\
\hline Yes & 130 & 14 & 1 & & & \\
\hline No & 166 & 28 & $0.64(0.32,1.26)$ & 0.197 & $1.70(0.80,3.61)$ & 0.168 \\
\hline \multicolumn{7}{|c|}{ Hyperthyroidism } \\
\hline Yes & 45 & 6 & 1 & & & \\
\hline No & 251 & 36 & $0.93(0.37,2.33)$ & 0.877 & & \\
\hline
\end{tabular}

BMI, body mass index; VTE, venous thromboembolism; concomitant drugs use included antiplatelets, statins, antihypertensives, antithyroid drugs, and antiretroviral drugs.

significant association with good TTR outcome (TTR $\geq 65 \%$ ). Global studies documented that old age, obese individuals, other concomitant drugs intake, excessive alcohol intake, and renal or hepatic dysfunctions were among the listed causes of poor TTR outcome (TTR $<65 \%)[8-11$, 17-21]. Direct oral anticoagulants (DOACs) are recently introduced anticoagulants, which have a number of advantages over warfarin, despite limited access, cost issues, and availability of antidote. DOACs are prescribed in fixed doses, have fewer interactions with food and drugs, and do not require routine anticoagulant monitoring. Their use might be considered for eligible patients with atrial fibrillation and venous thromboembolism $[9-11,14,16]$.

4.1. Limitation of the Study. Since it was a cross-sectional study, the determined anticoagulation level might not be a true reflection of what happens all the time. Generalizability to the study population was limited, since the nonprobability sampling method was used to recruit study subjects.

\section{Conclusion}

The quality of anticoagulation control with warfarin in the study subjects was very low. The authors recommend to implement a validated warfarin-dose titration protocol and to establish anticoagulation clinics to mitigate the low anticoagulation level.

\section{Abbreviations}

ACC: American College of Cardiology

AHA: American Heart Association

AOR: Adjusted odds ratio

ART: Antiretroviral therapy

CI: $\quad$ Confidence interval

COR: Crude odds ratio

DOACs: Direct oral anticoagulants

INR: International normalized ratio

IRB: Institutional Review Board

PTU: Propylthiouracil

TTR: Time spent within the therapeutic range

VTE: Venous thromboembolism.

\section{Data Availability}

All data generated and analyzed are included within this research article.

\section{Ethical Approval}

Ethical approval was obtained from the Institutional Review Board of College of Medicine and Health Sciences, University of Gondar. Formal letter of permission was obtained from the University of Gondar hospital administrative body.

\section{Consent}

Study subjects were recruited only after informed written consent was obtained. Written informed consent for publication was also obtained from study subjects.

\section{Disclosure}

A preprint has previously been published [32]. The funding body had no role in the design of the study, data collection, analysis and interpretation of the data.

\section{Conflicts of Interest}

The authors declare that they have no conflicts of interest.

\section{Authors' Contributions}

ZL contributed to the conception, design, data collection, analysis, writing, and review of the manuscript. AT contributed to the conception, design, analysis, writing, and review of the manuscript. NB and TT contributed to conception, design, analysis, and review of the manuscript. All authors read and approved the final manuscript and approved its submission for publication.

\section{Acknowledgments}

The authors are grateful to thank the study participants and their health personnel. Funding for the research was obtained from the "Research and Publication Office" of College of Medicine and Health Sciences, University of Gondar.

\section{References}

[1] C. T. January, L. S. Wann, J. S. Alpert, H. Calkins, J. E. Cigarroa, and J. B. Conti, "AHA/ACC/HRS guideline for the management of patients with atrial fibrillation: a report of the American college of cardiology/American heart association task force on practice guidelines and the heart rhythm society," Journal of American College Cardiology, vol. 64, no. 21, pp. 2246-2280, 2014. 
[2] T. L. Ortel, I. Neumann, W. Ageno et al., "American Society of hematology 2020 guidelines for management of venous thromboembolism: treatment of deep vein thrombosis and pulmonary embolism," Blood Advances, vol. 4, no. 99, pp. 4693-4738, 2020.

[3] J. Hirsh, V. Fuster, J. Ansell, and J. L. Halperin, "American heart association/American college of cardiology foundation guide to warfarin therapy," Circulation, vol. 107, no. 12, pp. 1692-1711, 2003.

[4] J. Hirsh, J. E. Dalen, D. R. Anderson et al., "Oral Anticoagulants: mechanism of action, clinical effectiveness, and optimal therapeutic range," Chest, vol. 119, no. 1, pp. 8S-21S, 2001.

[5] J. M. Rivera-Caravaca, V. Roldán, M. A. Esteve-Pastor et al., "Importance of time in therapeutic range on bleeding risk prediction using clinical risk scores in patients with atrial fibrillation," Scientific Reports, vol. 7, no. 1, Article ID 12066, 2017.

[6] P. M. G. Erkens, H. ten Cate, H. R. Büller, and M. H. Prins, "Benchmark for Time in therapeutic range in venous thromboembolism: a systematic review and meta-analysis," PLoS One, vol. 7, no. 9, Article ID e42269, 2012.

[7] D. E. Singer, A. S. Hellkamp, J. P. Piccini et al., "Impact of global geographic region on time in therapeutic range on warfarin anticoagulant therapy: data from the rocket AF clinical trial," Journal of the American Heart Association, vol. 2, Article ID e000067, 2013.

[8] A. J. Rose, A. Ozonoff, L. E. Henault, and E. M. Hylek, "Warfarin for atrial fibrillation in community-based practise," Journal of Thrombosis and Haemostasis, vol. 6, no. 10, pp. 1647-1654, 2008.

[9] A. F. Macedo, J. Bell, C. McCarron et al., "Determinants of oral anticoagulation control in new warfarin patients: analysis using data from Clinical practice research datalink," Thrombosis Research, vol. 136, no. 2, pp. 250-260, 2015.

[10] V. Barrios, C. Escobar, L. Prieto et al., "Anticoagulation control in patients with nonvalvular atrial fibrillation attended at primary care centers in Spain: the PAULA study," Revista Española de Cardiología (English Edition), vol. 68, no. 9, pp. 769-776, 2015.

[11] D. Caldeira, I. Cruz, G. Morgado et al., "Evaluation of time in therapeutic range in anticoagulated patients: a single-center, retrospective, observational study," BMC Research Notes, vol. 7, no. 1, pp. 891-895, 2014.

[12] J. C. Mwita, J. M. Francis, A. A. Oyekunle, M. Gaenamong, M. Goepamang, and M. G. M. D. Magafu, "Quality of anticoagulation with warfarin at a tertiary hospital in Botswana," Clinical and Applied Thrombosis/Hemostasis, vol. 24, no. 4, pp. 596-601, 2018.

[13] B. O. Sonuga, D. A. Hellenberg, C. S. Cupido, and C. Jaeger, "Profile and anticoagulation outcomes of patients on warfarin therapy in an urban hospital in Cape town, South Africa," African Journal of Primary Health care \& Family Medicine, vol. 8, no. 1, pp. e1-8, 2016.

[14] I. Ebrahim, A. Bryer, K. Cohen, J. P. Mouton, W. Msemburi, and M. Blockman, "Poor anticoagulation control in patients taking warfarin at a tertiary and district-level prothrombin clinic in Cape town, South Africa," South African Medical Journal, vol. 108, no. 6, pp. 490-494, 2018.

[15] T. G. Fenta, T. Assefa, and B. Alemayehu, "Quality of anticoagulation management with warfarin among outpatients in a tertiary hospital in Addis Ababa, Ethiopia: a retrospective cross-sectional study," BMC Health Services Research, vol. 17, no. 1, pp. 389-396, 2017.
[16] E. Endewunet, A. Tadesse, A. Adane, and M. Abdulkadir, "Appropriate use of anti-thrombotic therapy in patients with atrial fibrillation at single-center experience, Northwest Ethiopia," BMC Cardiovascular Disorders, vol. 20, no. 1, pp. 375-384, 2020.

[17] J. A. Roth, K. Bradley, K. E. Thummel, D. L. Veenstra, and D. Boudreau, "Alcohol misuse, genetics, and major bleeding among warfarin therapy patients in a community setting," Pharmacoepidemiology and Drug Safety, vol. 24, no. 6, pp. 619-627, 2015.

[18] G. Teklay, N. Shiferaw, B. Legesse, and M. Bekele, "Drug-drug interactions and risk of bleeding among inpatients on warfarin therapy: a prospective observational study," Thrombosis Journal, vol. 12, no. 1, pp. 20-28, 2014.

[19] N. A. Limdi, T. D. Nolin, S. L. Booth et al., "Influence of kidney function on risk of supratherapeutic international normalized ratio-related hemorrhage in warfarin users: a prospective cohort study," American Journal of Kidney Diseases, vol. 65, no. 5, pp. 701-709, 2015.

[20] A. Qamar, M. Vaduganathan, N. J. Greenberger, and R. P. Giugliano, "Oral anticoagulation in patients with liver disease," Journal of the American College of Cardiology, vol. 71, no. 19, pp. 2162-2175, 2018.

[21] R. Hart, D. L. Veenstra, D. M. Boudreau, and J. A. Roth, "Impact of body mass index and genetics on warfarin major bleeding outcomes in a community setting," The American Journal of Medicine, vol. 130, no. 2, pp. 222-228, 2017.

[22] D. A. Lane and G. Y. H. Lip, "Maintaining therapeutic anticoagulation," Chest, vol. 131, no. 5, pp. 1277-1279, 2007.

[23] L. Schmitt, J. Speckman, and J. Ansell, "Quality assessment of anticoagulation dose management: comparative evaluation of measures of time-in-therapeutic range," Journal of Thrombosis and Thrombolysis, vol. 15, no. 3, pp. 213-216, 2003.

[24] L. M. Hess, M. A. Raebel, D. A. Conner, and D. C. Malone, "Measurement of adherence in pharmacy administrative databases: a proposal for standard definitions and preferred measures," Annals of Pharmacotherapy, vol. 40, no. 7-8, pp. 1280-1288, 2006.

[25] S. Schulman and C. Kearon, "Definition of major bleeding in clinical investigations of antihemostatic medicinal products in non-surgical patients," Journal of Thrombosis and Haemostasis, vol. 3, no. 4, pp. 692-694, 2005.

[26] S. Kaatz, D. Ahmad, A. C. Spyropoulos, and S. Schulman, "Definition of clinically relevant non-major bleeding in studies of anticoagulants in atrial fibrillation and venous thromboembolic disease in non-surgical patients: communication from the SSC of the ISTH," Journal of Thrombosis and Haemostasis, vol. 13, no. 11, pp. 2119-2126, 2015.

[27] B. Crzymala-Lubanski, S. Sjalander, H. Renlund, P. J. Svensson, and A. Sjalander, "Computer aided warfarin dosing in the Swedish national quality registry auricula-algorithmic suggestions are promising better than manual changed doses," Thrombosis Research, vol. 131, pp. 130-134, 2013.

[28] D. M. Witt, N. P. Clark, S. Kaatz, T. Schnurr, and J. E. Ansell, "Guidance for the practical management of warfarin therapy in the treatment of venous thromboembolism," Journal of Thrombosis and Thrombolysis, vol. 41, no. 1, pp. 187-205, 2016.

[29] O. C. Melamed, G. Horowitz, A. Elhayany, and S. Vinker, "Quality of anticoagulation control among patients with atrial fibrillation," The American Journal of Managed Care, vol. 17, no. 3, pp. 232-237, 2011.

[30] G. Palareti, C. Legnani, G. Guazzaloca et al., "Risks factors for highly unstable response to oral anticoagulation: a case- 
control study," British Journal of Haematology, vol. 129, no. 1, pp. 72-78, 2005.

[31] W. Kudzi, S. Y. Ahorhorlu, B. Dzudzor, E. Olayemi, E. T. Nartey, and R. H. Asmah, "Genetic polymorphisms of patients on stable warfarin maintenance therapy in a Ghanaian population," BMC Research Notes, vol. 9, no. 1, pp. 507-514, 2016.

[32] Z. Liyew, A. Tadesse, N. Bekele, and T. Tsegaye, "Evaluation of anticoagulation outcome among patients taking warfarin in university of gondar hospital, northwest Ethiopia," https:// www.researchsquare.com/article/rs-138820/v1. 